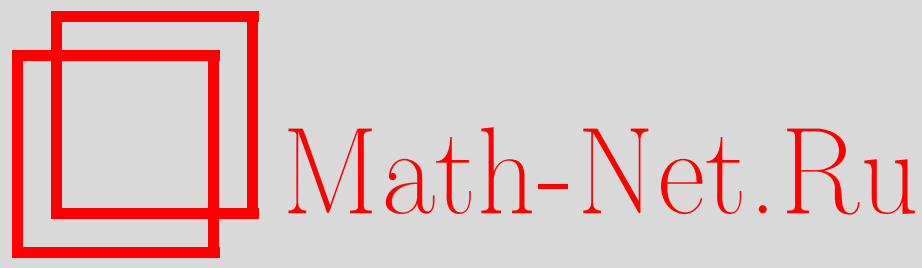

K. Сайеванд, К. Пичагхи, Повторный анализ открытой проблемы, связанной с дробным уравнением Шредингера, ТМФ, 2017, том 192, номер 1, 103-114

DOI: https://doi.org/10.4213/tmf9224

Использование Общероссийского математического портала Math-Net.Ru подразумевает, что вы прочитали и согласны с пользовательским соглашением http://www . mathnet.ru/rus/agreement

Параметры загрузки:

IP : 54.224 .187 .69

26 апреля 2023 г., 13:34:23

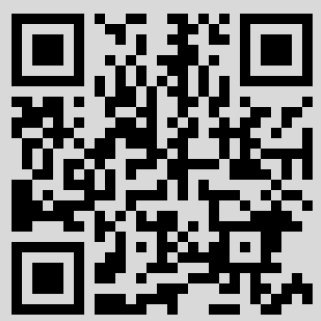




\title{
ПОВТОРНЫЙ АНАЛИЗ ОТКРЫТОЙ ПРОБЛЕМЫ, СВЯЗАННОЙ С ДРОБНЫМ УРАВНЕНИЕМ ШРЕДИНГЕРА
}

\begin{abstract}
Недавно было показано, что в методе построения решений, предложенном Ласкиным, встречаются некоторые сложности при нахождении собственных чисел и собственных векторов одномерного, не зависящего от времени дробного уравнения Шредингера с бесконечной потенциальной ямой, которая встречается в квантовой механике. Фактически эта проблема еще не решена. Предложен новый дробный подход, позволяющий преодолеть ограничения некоторых принципов, введенных ранее. При выводе решений используется метод, основанный на собственных функциях дробных производных Вейля. Решение получено в замкнутом и пригодном для расчета виде в терминах функций Миттаг-Леффлера и дробных тригонометрических функций. Оно представляет собой простое обобщение результатов, полученных ранее Ласкиным с соавторами.
\end{abstract}

Ключевые слова: дробное уравнение Шредингера, бесконечная потенциальная яма, дробная производная Рисса, функция Миттаг-Леффлера.

DOI: https://doi.org/10.4213/tmf9224

\section{1. ВВЕДЕНИЕ}

Фейнман и Гиббс [1] воссоздали уравнение Шредингера, они воспользовались подходом, основанным на интегралах по траекториям, рассматривая гауссово распределение вероятности. Этот подход был в дальнейшем обобщен Ласкиным [2], [3] в формулировке дробного уравнения Шредингера путем сведения фейнмановских интегралов по квантово-механическим траекториям типа броуновских к интегралам по траекториям типа Леви. Полученное таким образом уравнение Шредингера содержит дробные производные по пространству и времени.

Как и во многих других работах, в работах [4], [5] рассмотрено уравнение Шредингера с дробными производными по пространству, а также уравнение Шредингера с дробными производными как по пространству, так и по времени с использованием квантовой производной Рисса и некоторых специальных потенциальных полей,

*Faculty of Mathematical Sciences, University of Malayer, Malayer, Iran. E-mail: ksayehvand@malayeru.ac.ir, kazem.pichaghchi@stu.malayeru.ac.ir 
включая нулевой потенциал (свободная частица), дельта-потенциал, бесконечную потенциальную яму, потенциал Кулона и прямоугольный барьер. В своей недавней работе [6] Дзэн с соавторами предположили, что полученные кусочно-непрерывным способом решения уравнения Шредингера с дробной производной по пространству неверны. Их соображения основаны на противоречиях, возникающих при подстановке в пространственно-дробное уравнение Шредингера волновой функции основного состояния задачи с бесконечной потенциальной ямой. Они пришли к выводу, что многие известные в литературе точные решения неверны, за исключением решения, соответствующего потенциалу в виде дельта-функции. Это не только вызывает сомнения в справедливости известных в литературе решений, но также создает серьезные затруднения при выявлении полезных решений. В частности, Лучко [7] показал, что явные формулы для собственных чисел $E_{n}=D_{\alpha}(\pi \hbar n /(2 a))^{\alpha}, n \in \mathbb{N}$, и собственных функций

$$
\psi_{n=2 m-1}^{\text {even }}(x)=\left\{\begin{array}{ll}
A \cos \left(\frac{\pi n x}{2 a}\right), & |x|<a, \\
0, & |x| \geqslant a,
\end{array} \psi_{n=2 m}^{\text {odd }}(x)= \begin{cases}A \sin \left(\frac{\pi n x}{2 a}\right), & |x|<a, \\
0, & |x| \geqslant a\end{cases}\right.
$$

одномерного, не зависящего от времени дробного уравнения Шредингера

$$
\left(D_{\alpha}\left(-\hbar^{2} \Delta\right)^{\alpha / 2}+V(x)\right) \psi(x)=E \psi(x), \quad 1<\alpha<2, \quad x \in \mathbb{R}
$$

в случае бесконечной потенциальной ямы

$$
V(x)= \begin{cases}0, & |x|<a \\ \infty, & |x| \geqslant a\end{cases}
$$

полученные в работах [5], [8], а также во многих других работах различных авторов, не могут быть справедливыми. Действительно, в этих работах утверждается, что соответствующие дробному случаю решения для одномерной прямоугольной ямы совпадают с решениями для стандартного недробного случая, при этом изменяются только энергии. Оператор $\left(-\hbar^{2} \Delta\right)^{\alpha / 2}$ в уравнении (2) означает квантовую производную Рисса, которая определяется в разделе 2. Отметим, что в работе [6] рассмотрен случай $\alpha \in] 0,1[$, тогда как в настоящей работе мы сосредоточимся на случае $\alpha \in] 1,2[$ и применим совершенно другой метод. По-видимому, проблема определения правильных собственных чисел и собственных функций уравнения (2) с потенциалом (3) еще не решена (см. [9], раздел 2.10). Известными являются асимптотическое поведение и некоторые численные оценки собственных чисел (см., например, работы [10]-[12] и имеющиеся в них ссылки).

Отметим, что в недавних работах Байна [8], [13] была предпринята попытка опровергнуть суждения, представленные Дзэном с соавторами [6]. В частности, Байн попытался показать, что указанное в работе [6] противоречие можно преодолеть, вычисляя соответствующий интеграл надлежащим способом, и, следовательно, оно не означает несправедливость формул (1). В недавней работе Хавкинса и Шварца [14] подвергается критике предложенная в статьях Байна [8], [13] аргументация и утверждается, что результаты работы [6] остаются верными. 


\section{2. АНАЛИЗ ДРОБНОГО УРАВНЕНИЯ ШРЕДИНГЕРА С БЕСКОНЕЧНОЙ ПОТЕНЦИАЛЬНОЙ ЯМОЙ}

В настоящей работе изучается одномерное, не зависящее от времени дробное уравнение Шредингера порядка $1<\alpha<2$, которое имеет вид

$$
\left((-\Delta)^{\alpha / 2}+V(x)\right) \phi(x)=\lambda \phi(x), \quad x \in \mathbb{R} .
$$

Все величины в уравнении (4) считаются безразмерными, поэтому коэффициент перед квантовой производной Рисса, которая часто называется дробным лапласианом, можно положить равным единице без потери общности.

Для достаточно хорошо ведущей себя функции $f$ квантовая производная Рисса (дробный лапласиан) $(-\Delta)^{\alpha / 2}$ с $\alpha>0$ вводится как псевдодифференциальный оператор с символом $|\omega|^{\alpha}$

$$
\mathcal{F}\left\{(-\Delta)^{\alpha / 2} f(x), \omega\right\}=|\omega|^{\alpha} \int_{-\infty}^{\infty} e^{-i \omega x} f(x) d x, \quad \omega \in \mathbb{R},
$$

где $\mathcal{F}$ - преобразование Фурье функции $f$.

Квантовую производную Рисса при $0<\alpha<2$ можно представить в виде гиперсингулярного интеграла [7]

$$
(-\Delta)^{\alpha / 2} f(x)=\frac{1}{2 \Gamma(-\alpha) \cos (\alpha \pi / 2)} \int_{0}^{\infty} \frac{f(x-\xi)-2 f(x)+f(x+\xi)}{\xi^{1+\alpha}} d \xi .
$$

Другое представление квантовой производной Рисса, справедливое при $0<\alpha<2$, $\alpha \neq 1$, получено в работе [7] и имеет вид

$$
(-\Delta)^{\alpha / 2} f(x)=\frac{1}{2 \cos (\alpha \pi / 2)}\left(D_{+}^{\alpha}+D_{-}^{\alpha}\right) f(x),
$$

где $D_{+}^{\alpha}$ и $D_{-}^{\alpha}-$ соответственно лево- и правосторонние дробные производные Вейля порядка $\alpha$. Они определены для $x \in \mathbb{R}$ и $\alpha>0, n \leqslant \alpha<n+1, n \in \mathbb{N}$, следующим образом [7]:

$$
\left(D_{+}^{\alpha} f\right)(x):=\left(\frac{d}{d x}\right)^{n}\left(W_{+}^{n-\alpha} f\right)(x), \quad\left(D_{-}^{\alpha} f\right)(x):=\left(-\frac{d}{d x}\right)^{n}\left(W_{-}^{n-\alpha} f\right)(x),
$$

где $W_{ \pm}^{\alpha}-$ лево- и правосторонние дробные интегралы Вейля, которые при $\alpha>0$ вводятся по формулам

$$
\begin{aligned}
& \left(W_{+}^{\alpha} f\right)(x)=\frac{1}{\Gamma(\alpha)} \int_{-\infty}^{x}(x-\xi)^{\alpha-1} f(\xi) d \xi \\
& \left(W_{-}^{\alpha} f\right)(x)=\frac{1}{\Gamma(\alpha)} \int_{x}^{\infty}(\xi-x)^{\alpha-1} f(\xi) d \xi
\end{aligned}
$$

При $\alpha=0$ дробные интегралы Вейля определяются как тождественные операторы. Кроме того, определим дробный интеграл Римана-Лиувилля порядка $\alpha$ по формуле [15]-[17]

$$
\left(I_{a^{+}}^{\alpha} f\right)(x)=\frac{1}{\Gamma(\alpha)} \int_{a}^{x}(x-\xi)^{\alpha-1} f(\xi) d \xi, \quad \alpha>0
$$


Teopema 1. Пусть $a \in \mathbb{R}_{+}, \alpha, \beta, \lambda \in \mathbb{C}, \min \{\operatorname{Re} \alpha, \operatorname{Re} \beta\}>0$, mогдa $\partial л я x>a$ имеем

$$
I_{a^{+}}^{\lambda}\left[(t-a)^{\beta-1} E_{\alpha, \beta}\left[\omega(t-a)^{\alpha}\right]\right](x)=(x-a)^{\beta+\lambda-1} E_{\alpha, \beta+\lambda}\left[\omega(x-a)^{\alpha}\right] .
$$

ДокАЗАТЕЛЬСтво. Имеем

$$
I_{a^{+}}^{\lambda}\left[(t-a)^{\beta-1}\right](x)=\frac{\Gamma(\beta)}{\Gamma(\beta+\lambda)}(x-a)^{\beta+\lambda-1},
$$

далее

$$
\begin{aligned}
I_{a^{+}}^{\lambda}[(t & \left.-a)^{\beta-1} E_{\alpha, \beta}\left(\omega(t-a)^{\alpha}\right)\right](x)= \\
& =I_{a^{+}}^{\lambda}\left[\sum_{k=0}^{\infty} \frac{\omega^{k}(t-a)^{\alpha k+\beta-1}}{\Gamma(\alpha k+\beta)}\right](x)= \\
& =\sum_{k=0}^{\infty} \frac{\omega^{k}}{\Gamma(\alpha k+\beta)} \frac{\Gamma(\alpha k+\beta)}{\Gamma(\alpha k+\beta+\lambda)}(x-a)^{\alpha k+\beta+\lambda-1}= \\
& =(x-a)^{\beta+\lambda-1} E_{\alpha, \beta+\lambda}\left[\omega(x-a)^{\alpha}\right],
\end{aligned}
$$

что завершает доказательство.

Следует отметить, что $E_{\alpha, \beta}(z)$ при $\alpha>0, \beta>0$ является функцией Миттаг-Леффлера, для которой на всей комплексной плоскости справедливо представление в виде следующего ряда [18]:

$$
E_{\alpha, \beta}(z)=\sum_{k=0}^{\infty} \frac{z^{k}}{\Gamma(\alpha k+\beta)}, \quad z \in \mathbb{C} .
$$

Во всех последующих теоремах используются формулы Дирихле (теорема Фубини)

$$
\begin{aligned}
\int_{a}^{b} d x \int_{a}^{x} f(x, t) d t & =\int_{a}^{b} d t \int_{t}^{b} f(x, t) d x \\
\frac{d}{d x} \int_{a}^{x} h(x, t) d t & =\left[\int_{a}^{x} \frac{\partial}{\partial x} h(x, t) d t\right]+h(x, x) .
\end{aligned}
$$

Рассмотрим интегральный оператор, зависящий от дробного интегрального оператора Вейля и содержащий в своем ядре функцию $\mathrm{E}_{\alpha, \beta}(z)$ :

$$
\left(\mathrm{E}_{\alpha, \beta, \omega, \infty} \varphi\right)(\omega)=\int_{x}^{\infty}(t-x)^{\beta-1} E_{\alpha, \beta}\left[\omega(t-x)^{\alpha}\right] \varphi(t) d t .
$$

Теперь мы готовы сформулировать следующие теоремы.

Teоpema 2. Пусть $\alpha, \beta, \omega \in \mathbb{C}$, причем $\min \{\operatorname{Re} \alpha, \operatorname{Re} \beta\}>0$, тогда

$$
\left(W_{-}^{\lambda} \mathrm{E}_{\alpha, \beta, \omega, \infty} \varphi\right)(x)=\left(\mathrm{E}_{\alpha, \beta+\lambda, \omega, \infty} \varphi\right)(x)=\left(\mathrm{E}_{\alpha, \beta, \omega, \infty} W_{-}^{\lambda} \varphi\right)(x) .
$$


ДокАЗАТЕЛьство. Воспользовавшись определениями (9) и (16), а также формулой Дирихле (15), получим

$$
\begin{aligned}
\left(W_{-}^{\lambda} \mathrm{E}_{\alpha, \beta, \omega, \infty} \varphi\right)(x) & =\frac{1}{\Gamma(\lambda)}\left\{\int_{x}^{\infty}(u-x)^{\lambda-1}\left[\int_{u}^{\infty}(t-u)^{\beta-1} E_{\alpha, \beta}\left[\omega(t-u)^{\alpha}\right] \varphi(t) d t\right]\right\} d u= \\
& =\int_{x}^{\infty} \frac{1}{\Gamma(\lambda)}\left\{\int_{x}^{t}(u-x)^{\lambda-1}(t-u)^{\beta-1} E_{\alpha, \beta}\left[\omega(t-u)^{\alpha}\right] d u\right\} \varphi(t) d t .
\end{aligned}
$$

Пусть $\tau=(t-u)$, тогда

$$
\left(W_{-}^{\lambda} \mathrm{E}_{\alpha, \beta, \omega, \infty} \varphi\right)(x)=\int_{x}^{\infty} \frac{1}{\Gamma(\lambda)}\left[\int_{0}^{t-x}(t-x-\tau)^{\lambda-1} \tau^{\beta-1} E_{\alpha, \beta}\left(\omega \tau^{\alpha}\right) d t\right] \varphi(t) d t .
$$

Воспользовавшись формулой (10) и результатом теоремы 1 , получим

$$
\begin{aligned}
\left(W_{-}^{\lambda} \mathrm{E}_{\alpha, \beta, \omega, \infty} \varphi\right)(x) & =\int_{x}^{\infty} I_{0}^{\lambda}\left[\tau^{\beta-1} E_{\alpha, \beta}\left(\omega \tau^{\alpha}\right)\right](t-x) \varphi(t) d t= \\
& =\int_{x}^{\infty}(t-x)^{\beta+\lambda-1} E_{\alpha, \beta+\lambda}\left[\omega(t-x)^{\alpha}\right] \varphi(t) d t= \\
& =\left(\mathrm{E}_{\alpha, \beta+\lambda, \omega, \infty} \varphi\right)(x) .
\end{aligned}
$$

С другой стороны,

$$
\begin{aligned}
& \left(\mathrm{E}_{\alpha, \beta, \omega, \infty} W_{-}^{\lambda} \varphi\right)(x)= \\
& \quad=\int_{x}^{\infty}(t-x)^{\beta-1} E_{\alpha, \beta}\left[\omega(t-x)^{\alpha}\right] \frac{1}{\Gamma(\lambda)}\left[\int_{t}^{\infty}(u-t)^{\lambda-1} \varphi(u) d u\right] d t= \\
& \quad=\int_{x}^{\infty} \frac{1}{\Gamma(\lambda)}\left\{\int_{x}^{u}(t-x)^{\beta-1}(u-t)^{\lambda-1} E_{\alpha, \beta}\left[\omega(t-x)^{\alpha}\right] d t\right\} \varphi(u) d u .
\end{aligned}
$$

Пусть $\tau=t-x$, тогда получим

$$
\left(\mathrm{E}_{\alpha, \beta, \omega, \infty} W_{-}^{\lambda} \varphi\right)(x)=\int_{x}^{\infty} \frac{1}{\Gamma(\lambda)}\left[\int_{0}^{u-x}(u-x-\tau)^{\lambda-1} \tau^{\beta-1} E_{\alpha, \beta}\left(\omega \tau^{\alpha}\right) d \tau\right] \varphi(u) d u .
$$

Возвращаясь к формуле (10) и теореме 1, имеем

$$
\begin{aligned}
\left(\mathrm{E}_{\alpha, \beta, \omega, \infty} W_{-}^{\lambda} \varphi\right)(x) & =\int_{x}^{\infty} I_{0}^{\lambda}\left[\tau^{\beta-1} E_{\alpha, \beta}\left(\omega \tau^{\alpha}\right)\right](u-x) \varphi(u) d u= \\
& =\int_{x}^{\infty}(u-x)^{\beta+\lambda-1} E_{\alpha, \beta+\lambda}\left[\omega(u-x)^{\alpha}\right] \varphi(u) d u= \\
& =\left(\mathrm{E}_{\alpha, \beta+\lambda, \omega, \infty} \varphi\right)(x),
\end{aligned}
$$

что завершает доказательство.

Аналогичный результат для вейлевского дробного дифференцирования сформулирован в следующей теореме.

ТЕОРема 3. Если условие теоремы 2 выполнено, то

$$
\left(D_{-}^{\lambda} \mathrm{E}_{\alpha, \beta, \omega, \infty} \varphi\right)(x)=\left(\mathrm{E}_{\alpha, \beta-\lambda, \omega, \infty} \varphi\right)(x) .
$$


ДокАЗАТЕЛЬСТво. Воспользовавшись формулами (8), получим

$$
\left(D_{-}^{\lambda} \mathrm{E}_{\alpha, \beta, \omega, \infty} \varphi\right)(x)=(-1)^{n}\left(\frac{d}{d x}\right)^{n}\left(W_{-}^{n-\lambda} \mathrm{E}_{\alpha, \beta, \omega, \infty} \varphi\right)(x),
$$

откуда, применив теорему 2 , имеем

$$
\left(D_{-}^{\lambda} \mathrm{E}_{\alpha, \beta, \omega, \infty} \varphi\right)(x)=(-1)^{n}\left(\frac{d}{d x}\right)^{n} \int_{x}^{\infty}(t-x)^{\beta+n-\lambda-1} E_{\alpha, \beta-n-\lambda}\left[\omega(t-x)^{\alpha}\right] \varphi(t) d t .
$$

С помощью формулы Дирихле (15) получим

$$
\begin{aligned}
& \left(D_{-}^{\lambda} \mathrm{E}_{\alpha, \beta, \omega, \infty} \varphi\right)(x)=(-1)^{n}\left(\frac{d}{d x}\right)^{n-1} \times \\
& \quad \times\left(\int_{x}^{\infty} \frac{\partial}{\partial x}(t-x)^{\beta+n-\lambda-1} E_{\alpha, \beta+n-\lambda}\left[\omega(t-x)^{\alpha}\right] \varphi(t) d t\right)+ \\
& \quad+\lim _{t \rightarrow x^{+}}(t-x)^{\beta+n-\lambda-1} E_{\alpha, \beta+n-\lambda}\left[\omega(t-x)^{\alpha}\right] \varphi(t)= \\
& =(-1)^{n}\left(\frac{d}{d x}\right)^{n-1} \int_{x}^{\infty} \sum_{k=0}^{\infty} \frac{\omega^{k}(\alpha k+\beta+n-\lambda-1)}{\Gamma(\alpha k+\beta+n-\lambda)}(t-x)^{\alpha k+\beta+n-\lambda-2} \varphi(t) d t= \\
& =(-1)^{n}\left(\frac{d}{d x}\right)^{n-1} \int_{x}^{\infty}(-1)(t-x)^{\beta+n-\lambda-2} E_{\alpha, \beta+n-\lambda-1}\left[\omega(t-x)^{\alpha}\right] \varphi(t) d t .
\end{aligned}
$$

Повторяя эти вычисления $n-1$ раз, приходим к следующему результату:

$$
\begin{aligned}
\left(D_{-}^{\lambda} \mathrm{E}_{\alpha, \beta, \omega, \infty} \varphi\right)(x) & =(-1)^{n}(-1)^{n} \int_{x}^{\infty}(t-x)^{\beta-\lambda-1} E_{\alpha, \beta-\lambda}\left[\omega(t-x)^{\alpha}\right] \varphi(t) d t= \\
& =\left(\mathrm{E}_{\alpha, \beta-\lambda, \omega, \infty} \varphi\right)(x),
\end{aligned}
$$

что завершает доказательство.

Как показано в приведенных ниже теоремах, величины

$$
\int_{x}^{\infty}(t-x)^{\alpha-1} E_{\alpha, \alpha}\left[\alpha(i \omega(t-x))^{\alpha}\right] E_{\alpha, \alpha}\left((i \omega t)^{\alpha}\right) d t=\mathrm{C}|\omega|^{-\alpha} E_{\alpha, \alpha}\left((i \omega x)^{\alpha}\right),
$$

где C - постоянная, являются собственными функциями лево- и правосторонних дробных производных Вейля порядка $\alpha, \alpha>0$.

Teоpema 4. Пусть $\omega \in \mathbb{R}, \omega \neq 0$ u $n<\alpha<n+1$. Тогда $a^{1)}$

$$
\begin{aligned}
& D_{-}^{\alpha}\left[\int_{\xi}^{\infty}(t-\xi)^{\alpha-1} E_{\alpha, \alpha}\left[\alpha(i \omega(t-\xi))^{\alpha}\right] E_{\alpha, \alpha}\left((i \omega t)^{\alpha}\right) d t\right](x)= \\
& =i^{\alpha} \operatorname{sgn}(\omega) \Gamma(1+\alpha)(1+\alpha)^{-1} \times \\
& \quad \times E_{\alpha, \alpha}\left((i \omega x)^{\alpha}\right)\left[\cos _{\alpha}\left((\alpha \pi / 2)^{\alpha}\right)+\operatorname{sgn}(\omega) \sin _{\alpha}\left((\alpha \pi / 2)^{\alpha}\right)\right] .
\end{aligned}
$$

1)Через $i^{\alpha}$ обозначена дробная мнимая единица (см. более подробное изложение в работах [19], [20]). 
ДокАЗАТЕЛЬСтво. Из теоремы 3 следует равенство

$$
\begin{gathered}
D_{-}^{\alpha}\left[\int_{\xi}^{\infty}(t-\xi)^{\alpha-1} E_{\alpha, \alpha}\left[\alpha(i \omega(t-\xi))^{\alpha}\right] E_{\alpha, \alpha}\left((i \omega t)^{\alpha}\right) d t\right](x)= \\
=\int_{x}^{\infty}(t-x)^{-1} E_{\alpha, 0}\left[\alpha(i \omega(t-x))^{\alpha}\right] E_{\alpha, \alpha}\left((i \omega t)^{\alpha}\right) d t .
\end{gathered}
$$

Теперь воспользуемся формулой

$$
E_{\alpha, \alpha}\left(\lambda t^{\alpha}\right)=\frac{1}{\lambda t^{\alpha}} E_{\alpha, 0}\left(\lambda t^{\alpha}\right)
$$

Следовательно, получим

$$
\begin{aligned}
& \int_{x}^{\infty}(t-x)^{-1} E_{\alpha, 0}\left[\alpha(i \omega(t-x))^{\alpha}\right] E_{\alpha, \alpha}\left((i \omega t)^{\alpha}\right) d t= \\
& =i^{\alpha} \operatorname{sign}(\omega) \Gamma(1+\alpha)(1+\alpha)^{-1} \times \\
& \quad \times E_{\alpha, \alpha}\left((i \omega x)^{\alpha}\right)\left[\cos _{\alpha}\left((\alpha \pi / 2)^{\alpha}\right)+\operatorname{sign}(\omega) \sin _{\alpha}((\alpha \pi / 2))^{\alpha}\right] .
\end{aligned}
$$

Аналогичным способом можно доказать следующую теорему.

Teоpema 5. Пусть $\omega \in \mathbb{R}, \omega \neq 0 u n<\alpha<n+1$. Тогдa

$$
\begin{aligned}
& D_{+}^{\alpha}\left[\int_{\xi}^{\infty}(t-\xi)^{\alpha-1} E_{\alpha, \alpha}\left[\alpha(i \omega(t-\xi))^{\alpha}\right] E_{\alpha, \alpha}\left((i \omega t)^{\alpha}\right) d t\right](x)= \\
& =i^{\alpha} \operatorname{sign}(\omega) \Gamma(1+\alpha)(1+\alpha)^{-1} \times \\
& \quad \times E_{\alpha, \alpha}\left((i \omega x)^{\alpha}\right)\left[\cos _{\alpha}\left((\alpha \pi / 2)^{\alpha}\right)-\operatorname{sign}(\omega) \sin _{\alpha}\left((\alpha \pi / 2)^{\alpha}\right)\right] .
\end{aligned}
$$

Можно легко проверить, что

$$
E_{\alpha, \alpha}\left((i \omega x)^{\alpha}\right):=\cos _{\alpha}\left((\omega x)^{\alpha}\right)+\sin _{\alpha}\left((\omega x)^{\alpha}\right),
$$

где дробные тригонометрические функции введены по формулам

$$
\cos _{\alpha}\left((\omega x)^{\alpha}\right):=\sum_{k=0}^{\infty} \frac{(i \omega x)^{2 k \alpha}}{\Gamma[(2 k+1) \alpha]}, \quad \sin _{\alpha}\left((\omega x)^{\alpha}\right):=\sum_{k=0}^{\infty} \frac{(i \omega x)^{(2 k+1) \alpha}}{\Gamma[(k+1) 2 \alpha]} .
$$

Эти формулы вместе с представлением (7) квантовой производной Рисса приводят к уравнениям

$$
(-\Delta)^{\alpha / 2}\left\{\begin{array}{l}
\cos _{\alpha}\left((\omega x)^{\alpha}\right) \\
\sin _{\alpha}\left((\omega x)^{\alpha}\right)
\end{array}=\frac{|\omega|^{\alpha}}{\cos (\alpha \pi / 2)}\left\{\begin{array}{l}
\cos _{\alpha}\left((\omega x)^{\alpha}\right), \\
\sin _{\alpha}\left((\omega x)^{\alpha}\right),
\end{array}\right.\right.
$$

которые справедливы для $\omega \neq 0$ и $0<\alpha \leqslant 2, \alpha \neq 1$, и означают, что для $\omega \neq 0$ функции $\cos _{\alpha}\left((\omega x)^{\alpha}\right), \sin _{\alpha}\left((\omega x)^{\alpha}\right)$ являются собственными функциями квантовой производной Рисса с собственными значениями $|\omega|^{\alpha}$.

Теперь мы готовы представить собственные числа и собственные функции одномерного, не зависящего от времени дробного уравнения Шредингера (4) для случая 
бесконечной потенциальной ямы (3). Собственные значения $\lambda_{n}$ и собственные функции $\phi_{n}$ для задачи на собственные значения (4) с потенциалом (3) можно получить следующим образом.

Если волновая функция ф удовлетворяет уравнению (4) с потенциалом (3), она должна равняться нулю при $|x| \geqslant a$, т. е. волновую функцию $\phi$ можно представить в виде

$$
\phi(x)= \begin{cases}\varphi(x), & |x|<a, \\ 0, & |x| \geqslant a,\end{cases}
$$

с неизвестной функцией $\varphi$. Теперь уравнение

$$
(-\Delta)^{\alpha / 2} \phi(x)=\lambda \phi(x), \quad x \in \mathbb{R}
$$

нужно решить для функции ф вида (37). Эта задача эквивалентна следующей задаче на собственные значения:

$$
(-\Delta)^{\alpha / 2} \phi(x)=\lambda \phi(x), \quad \phi(-a)=\phi(a)=0 .
$$

Решение задачи (39) имеет вид

$$
\phi(x)=E_{\alpha, \alpha}\left((i \omega x)^{\alpha}\right) \quad \text { или } \quad \phi(x)=A \sin _{\alpha}\left((\omega x)^{\alpha}\right)+B \cos _{\alpha}\left((\omega x)^{\alpha}\right) .
$$

Из граничного условия $\phi(-a)=\phi(a)=0$ следуют уравнения

$$
A \sin _{\alpha}\left((\omega a)^{\alpha}\right)=0, \quad B \cos _{\alpha}\left((\omega a)^{\alpha}\right)=0 .
$$

Таким образом, решения распадаются на два класса: решения, в которых $A=0$, и решения, в которых $B=0$.

Поэтому собственные значения выражаются формулой $\lambda_{n}=(\alpha n \pi / 2 a)^{\alpha}, n \in \mathbb{N}$, а собственные функции $\phi_{n}$ приобретают следующий вид:

$$
\begin{array}{ll}
n=2 m-1: & \phi_{n}(x)=\left\{\begin{array}{ll}
A \cos _{\alpha}\left(\left(\frac{\alpha n \pi x}{2 a}\right)^{\alpha}\right), & |x|<a, \\
0, & |x| \geqslant a,
\end{array} \quad m \in \mathbb{N} .\right. \\
n=2 m: & \phi_{n}(x)= \begin{cases}A \sin _{\alpha}\left(\left(\frac{\alpha n \pi x}{2 a}\right)^{\alpha}\right), & |x|<a, \\
0, & |x| \geqslant a,\end{cases}
\end{array}
$$

Мы видим, что наши результаты, изображенные на рис. 1, находятся в хорошем согласии с приближенными решениями, полученными в работах [10]-[12].

Чтобы показать противоречивость решений (1), Дзэн с соавторами [6] остановились на основном состоянии с $n=1$ и сформулировали утверждение о том, что это решение, хотя и удовлетворяет граничным условиям $\psi_{1}(a)=\psi_{1}(-a)=0$, после подстановки в пространственное дробное уравнение Шредингера (39) приводит к противоречию. Мы проанализировали эту проблему и показали, что точное и правильное обращение с соответствующим интегралом, связанным с функциями (42), доказывает обратное. 


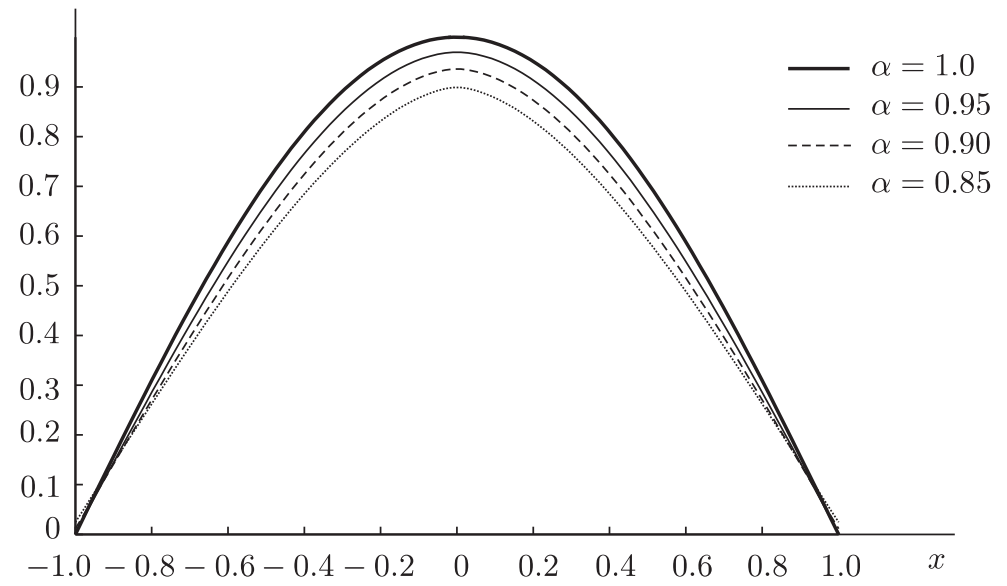

Рис. 1. График аппроксимации $\phi$ при $n=1$ и $a=1$.

Представление Фурье функции $\phi_{1}$ можно записать в виде

$$
\phi_{1}(\omega)=2 A\left(\frac{\alpha \pi}{2 a i^{2}}\right)^{\alpha} \frac{\cos _{\alpha}\left((a \omega)^{\alpha}\right)}{\omega^{2 \alpha}-(\alpha \pi / 2 a)^{2 \alpha}}, \quad|x|<a
$$

при подстановке в уравнение (39) оно приводит к функции $\psi_{1}(x)$ в виде интеграла:

$$
\psi_{1}(x)=\frac{A}{\pi \lambda_{1}}\left(\frac{\alpha \pi}{2 a i^{2}}\right)^{\alpha} \int_{-\infty}^{\infty} d \omega\left(\frac{2 a}{\alpha \pi}\right)^{2 \alpha} \frac{|\omega|^{\alpha} \cos _{\alpha}\left((a \omega)^{\alpha}\right)}{(2 a \omega / \alpha \pi)^{2 \alpha}-1} e^{i \omega x}, \quad|x|<a .
$$

Вводя новую переменную $\chi=(2 a / \alpha \pi) \omega$, этот интеграл можно переписать в виде

$$
\psi_{1}(x)=\frac{A \alpha}{2 a \lambda_{1} i^{2 \alpha}} \int_{-\infty}^{\infty} d \chi \frac{|\chi|^{\alpha} \cos _{\alpha}\left((\alpha \pi \chi / 2)^{\alpha}\right)}{\chi^{2 \alpha}-1} e^{i \alpha \pi \chi x / 2 a}, \quad|x|<a .
$$

Теперь докажем, что полученное выражение удовлетворяет граничным условиям при $x \rightarrow \pm a$. Заметим, что интеграл

$$
I=\int_{-\infty}^{\infty} d \chi \frac{|\chi|^{\alpha} \cos _{\alpha}\left((\alpha \pi \chi / 2)^{\alpha}\right)}{\chi^{2 \alpha}-1} e^{i \alpha \pi \chi x / 2 a}
$$

является сингулярным с полюсом на действительной оси в точке $\chi^{\alpha}= \pm 1$, этот интеграл можно вычислить с помощью аналитического продолжения как интеграл Коши в смысле главного значения (см. раздел 13 в [21]).

Подставляя

$$
\cos _{\alpha}\left((\alpha \pi \chi / 2)^{\alpha}\right)=\frac{1}{2}\left[E_{\alpha, \alpha}\left((i \alpha \pi \chi / 2)^{\alpha}\right)+E_{\alpha, \alpha}\left(-(i \alpha \pi \chi / 2)^{\alpha}\right)\right],
$$


напишем сначала этот интеграл в виде суммы двух интегралов:

$$
\begin{aligned}
I= & \frac{1}{2} \int_{-\infty}^{\infty} d \chi \frac{|\chi|^{\alpha} E_{\alpha, \alpha}\left((i \alpha \pi \chi / 2)^{\alpha}\right)}{\chi^{2 \alpha}-1} e^{i \alpha \pi \chi x / 2 a}+ \\
& +\frac{1}{2} \int_{-\infty}^{\infty} d \chi \frac{|\chi|^{\alpha} E_{\alpha, \alpha}\left(-(i \alpha \pi \chi / 2)^{\alpha}\right)}{\chi^{2 \alpha}-1} e^{i \alpha \pi \chi x / 2 a}= \\
= & \frac{(i \alpha \pi / 2 a)^{\alpha-1}}{2} \int_{-\infty}^{\infty} d \chi \frac{|\chi|^{\alpha} E_{\alpha, \alpha}\left((i \alpha \pi \chi / 2)^{\alpha}\right)}{\chi^{2 \alpha}-1} E_{\alpha, \alpha}\left((i \alpha \pi \chi x / 2 a)^{\alpha}\right)+ \\
& \quad+\frac{(i \alpha \pi / 2 a)^{\alpha-1}}{2} \int_{-\infty}^{\infty} d \chi \frac{|\chi|^{\alpha} E_{\alpha, \alpha}\left(-(i \alpha \pi \chi / 2)^{\alpha}\right)}{\chi^{2 \alpha}-1} E_{\alpha, \alpha}\left((i \alpha \pi \chi x / 2 a)^{\alpha}\right)= \\
= & I_{1}+I_{2} .
\end{aligned}
$$

Для вычисления первого интеграла $I_{1}$ замкнем контур интегрирования в верхней $\chi$-полуплоскости полуокружностью радиуса $R$ и затем обойдем полюс на действительной оси в верхней $\chi$-полуплоскости по малой полуокружности радиуса $\delta$. Согласно лемме Жордана в пределе при $R \rightarrow \infty$ вклад от большой полуокружности исчезает, что позволяет нам вычислить этот интеграл как интеграл Коши в смысле главного значения в пределе $\delta \rightarrow 0$ следующим образом (ср. [21], раздел 13):

$$
\begin{aligned}
\operatorname{PV}\left(I_{1}\right) & =\left(\frac{i \alpha \pi}{2 a}\right)^{\alpha-1}(i \pi)\left(\frac{i^{\alpha}}{2} \cos _{\alpha}\left(\left(\frac{\alpha \pi x}{2 a}\right)^{\alpha}\right)\right)= \\
& =\frac{a i^{2 \alpha}}{\alpha}\left(\frac{\alpha \pi}{2 a}\right)^{\alpha} \cos _{\alpha}\left(\left(\frac{\alpha \pi x}{2 a}\right)^{\alpha}\right) .
\end{aligned}
$$

Аналогично для вычисления $I_{2}$ замкнем контур, на этот раз в нижней $\chi$-полуплоскости, обойдем полюс по окружности в нижней $\chi$-полуплоскости и получим

$$
\operatorname{PV}\left(I_{2}\right)=-\left(\frac{i \alpha \pi}{2 a}\right)^{\alpha-1} i \pi\left[-\frac{i^{\alpha}}{2} \cos _{\alpha}\left(\left(\frac{\alpha \pi x}{2 a}\right)^{\alpha}\right)\right] .
$$

Складывая, получим главное значение интеграла $I$ в смысле Коши:

$$
\mathrm{PV}(I)=\frac{2 a}{\alpha} i^{2 \alpha}\left(\frac{\alpha \pi}{2 a}\right)^{\alpha} \cos _{\alpha}\left(\left(\frac{\alpha \pi x}{2 a}\right)^{\alpha}\right)
$$

которое, в отличие от результата Дзэна с соавторами [6], исчезает на границе при $x \rightarrow \pm a$. При подстановке найденных выражений в формулу (45) получим

$$
\psi_{1}(x)=\frac{A}{\lambda_{1}}\left(\frac{\alpha \pi}{2 a}\right)^{\alpha} \cos _{\alpha}\left(\left(\frac{\alpha \pi x}{2 a}\right)^{\alpha}\right), \quad|x|<a .
$$

Поскольку собственное значение основного состояния имеет вид

$$
\lambda_{1}=\left(\frac{\alpha \pi}{2 a}\right)^{\alpha}
$$

мы снова получим волновую функцию основного состояния

$$
\psi_{1}(x)=A \cos _{\alpha}\left(\left(\frac{\alpha \pi x}{2 a}\right)^{\alpha}\right), \quad|x|<a,
$$


которая удовлетворяет граничным условиям и, следовательно, противоречий не возникает.

Доказательство для произвольного $n$ можно получить по той же схеме. Например, для нечетных значений $n$ выражение (45) следует заменить на следующее соотношение:

$$
\psi_{n}(x)=\frac{A \alpha}{2 a \lambda_{n} i^{2 \alpha}} \sin _{\alpha}\left(\left(\frac{\alpha n \pi}{2}\right)^{\alpha}\right) \int_{-\infty}^{\infty} d \chi \frac{|\chi|^{\alpha} \cos _{\alpha}\left((\alpha n \pi \chi / 2)^{\alpha}\right)}{\chi^{2 \alpha}-1} e^{i \alpha n \pi \chi x / 2 a} .
$$

Теперь главное значение в смысле Коши интеграла

$$
I=\int_{-\infty}^{\infty} d \chi \frac{|\chi|^{\alpha} \cos _{\alpha}\left((\alpha n \pi \chi / 2)^{\alpha}\right)}{\chi^{2 \alpha}-1} e^{i \alpha n \pi \chi x / 2 a}
$$

найдем в виде

$$
\mathrm{PV}(I)=\frac{2 a}{\alpha} i^{2 \alpha} \sin _{\alpha}\left(\left(\frac{\alpha n \pi}{2}\right)^{\alpha}\right)\left(\frac{\alpha n \pi}{2}\right)^{\alpha} \cos _{\alpha}\left(\left(\frac{\alpha n \pi x}{2}\right)^{\alpha}\right),
$$

что при подстановке в формулу (55) приводит к выражению

$$
\begin{aligned}
\psi_{n}(x) & =\frac{A}{\lambda_{n}} \sin _{\alpha}^{2}\left(\left(\frac{\alpha n \pi}{2}\right)^{\alpha}\right)\left(\frac{\alpha n \pi}{2 a}\right)^{\alpha} \cos _{\alpha}\left(\left(\frac{\alpha n \pi x}{2 a}\right)^{\alpha}\right)= \\
& =A \cos _{\alpha}\left(\left(\frac{\alpha n \pi x}{2 a}\right)^{\alpha}\right), \quad n=1,3,5, \ldots
\end{aligned}
$$

Снова противоречий не возникает. Доказательство для четных значений $n$ аналогично.

\section{3. ЗАКЛЮЧЕНИЕ}

В цикле работ Ласкина [2], [3], [22], как и во многих других работах различных авторов, рассматривается пространственное дробное уравнение Шредингера с квантовой производной Рисса. В этих работах, в частности, утверждается, что получены явные формулы для собственных значений и собственных функций одномерного, не зависящего от времени дробного уравнения Шредингера с бесконечной потенциальной ямой. Результаты работ [6], [7] демонстрируют наличие определенных трудностей в предложенном Ласкиным методе построения решений при нахождении собственных значений и собственных функций упомянутого уравнения. Мы провели дополнительный анализ этой спорной проблемы и предложили новый дробный подход для описания собственных значений и собственных функций одномерного, не зависящего от времени дробного уравнения Шредингера для свободной частицы и для бесконечной прямоугольной потенциальной ямы, которые встречаются в квантовой механике. При выводе решений мы следовали методу, основанному на собственной функции дробной производной Вейля, при этом решение получено в замкнутой, пригодной для расчетов форме, выраженной через функции Миттаг-Леффлера и дробные тригонометрические функции. Основной результат получен в виде теоремы 3. Два частных случая этого результата даны в последующих теоремах. 


\section{Список литературы}

[1] R.P. Feynman, A. R. Hibbs, Quantum Mechanics and Path Integrals, McGraw-Hill, New York, 1965.

[2] N. Laskin, "Fractional quantum mechanics and Lévy path integrals", Phys. Lett. A, 268:4-6 (2000), 298-305.

[3] N. Laskin, "Fractional Schrödinger equation", Phys. Rev. E, 66:5 (2002), 056108, 7 pp.

[4] S. Wang, M. Xu, "Generalized fractional Schrödinger equation with space-time fractional derivatives", J. Math. Phys., 48:4 (2007), 043502, 10 pp.

[5] J. Dong, M. Xu, "Space-time fractional Schrödinger equation with time-independent potentials", J. Math. Anal. Appl., 344:2 (2008), 1005-1017.

[6] M. Jeng, S. L. Y. Xu, E. Hawkins, J. M. Schwarz, "On the nonlocality of the fractional Schrödinger equation", J. Math. Phys., 51:6 (2010), 062102, 6 pp.

[7] Y. Luchko, "Fractional Schrödinger equation for a particle moving in a potential well", J. Math. Phys., 54:1 (2013), 012111, 10 pp.

[8] S.S. Bayin, "On the consistency of the solutions of the space fractional Schrödinger equation", J. Math. Phys., 53:4 (2012), 042105, 9 pp.

[9] J. T. Machado, F. Mainardi, V. Kiryakova, "Fractional calculus: quo vadimus? (Where are we going?)", Fract. Calc. Appl. Anal., 18:2 (2015), 495-526.

[10] M. Kwaśnicki, "Eigenvalues of the fractional Laplace operator in the interval", J. Funct. Anal., 262:5 (2012), 2379-2402.

[11] A. Zoia, A. Rosso, M. Kardar, "Fractional Laplacian in bounded domains", Phys. Rev. E, 76:2 (2007), $11 \mathrm{pp}$.

[12] R. Herrmann, "The fractional Schrödinger equation and the infinite potential well-numerical results using the Riesz derivative", Gam. Ori. Chron. Phys., 1 (2013), 1-12, arXiv: 1210.4410.

[13] S. Bayin, "On the consistency of the solutions of the space fractional Schrödinger equation", J. Math. Phys., 53:4 (2012), 042105, 9 pp.

[14] E. Hawkins, J. M. Schwarz, "Comment on 'On the consistency of solutions of the space fractional Schrödinger equation"”, J. Math. Phys., 54:1 (2013), 014101, 5 pp.

[15] A. Kilbas, H. M. Srivastava, J. J. Trujillo, Theory and Applications of Fractional Differential Equations, North-Holland Mathematics Studies, 204, Elsevier, Amsterdam, 2006.

[16] D. Băleanu, O. G. Mustafa, D. O'Regan, "A Kamenev-type oscillation result for a linear $(1+\alpha)$-order fractional differential equation", Appl. Math. Comput., 259 (2015), 374-378.

[17] M. Al-Refai, Y. Luchko, "Maximum principle for the multi-term time-fractional diffusion equations with the Riemann-Liouville fractional derivatives", Appl. Math. Comput., 257 (2015), 40-51.

[18] K. Sayevand, K. Pichaghchi, "Successive approximation: a survey on stable manifold of fractional differential systems", Fract. Calc. Appl. Anal., 18:3 (2015), 621-641.

[19] X. J. Yang, "Local fractional integral transforms", Progr. Nonlinear Sci., 4 (2011), 1-225.

[20] X. J. Yang, Local Fractional Functional Analysis and Its Applications, Asian Academic Publ., Hong Kong, 2011.

[21] Ş. S. Bayin, Mathematical Methods in Science and Engineering, John Wiley and Sons, Hoboken, NJ, 2006.

[22] N. Laskin, "Fractals and quantum mechanics", Chaos, 10:4 (2000), 780-790. 